\title{
Tomography of high harmonic generation in a cluster jet
}

\author{
Chih-Hao Pai \\ Department of Physics, National Taiwan University, Taipei 106, Taiwan
}

Cheng-Cheng Kuo, Ming-Wei Lin, Jyhpyng Wang, and Szu-yuan Chen
Institute of Atomic and Molecular Sciences, Academia Sinica, Taipei 106, Taiwan

Jiunn-Yuan Lin

Department of Physics, National Chung Cheng University, Chia-Yi 621, Taiwan

\begin{abstract}
Received December 9, 2005; accepted December 21, 2005; posted January 4, 2006 (Doc. ID 66558)
Tomographic measurement of high harmonic generation in a cluster jet was demonstrated by programming the cluster density distribution with a laser machining technique. The growth of harmonic energy with the propagation of the pump pulse was resolved by scanning the end of the argon cluster distribution in the path of the pump pulse. A downstream shift of the position of rapid growth and a decrease of the slope with increasing backing pressure as results of changes in the phase-matching condition were observed, which explains the presence of an optimal backing pressure. (C) 2006 Optical Society of America
\end{abstract}

OCIS codes: $190.2620,220.4610,110.6960$.

High-order harmonic generation based on opticalfield ionization of atoms is an active field of research since it is a promising approach for the production of coherent short x-ray pulses that has widespread potential applications. ${ }^{1}$ The highest-order harmonic reported reached a $1 \mathrm{~nm}$ wavelength, ${ }^{2}$ and the shortest pulse duration reached the subfemtosecond time scale. ${ }^{3}$

To extend the practical application of high harmonic generation it is imperative to produce high harmonics with a shorter wavelength and higher energy conversion efficiency. Many methods for achieving this goal have been reported or proposed. Among them the use of an atomic cluster jet is considered to be a promising scheme. High harmonic generation in a cluster jet has been reported by Donnelly et $a l^{4}{ }^{4}$ and others. ${ }^{5,6}$ The observed harmonic cutoff energy for clusters shows an extension with respect to that for monomers. This extension and an associated increase in conversion efficiency were ascribed to the recombination of dissociated electrons with ions that are different from the parent ones in a cluster. ${ }^{7-9}$ Furthermore, it was proposed that the characteristic index of refraction of bulk nanoplasma gas can be used to attain phase matching of high harmonic generation under the condition of a high ionization level, ${ }^{10,11}$ which may lead to a dramatic increase of harmonic order and intensity. To date, all the reported experimental investigations on high harmonic generation from clusters have focused on the dependence of harmonic intensity on the microscopic properties of clusters. The growth of harmonics with laser beam propagation in a bulk cluster jet has not been studied experimentally. Such experiments may help us characterize the macroscopic effects, which is important for an understanding of the harmonic generation process and its optimization.

Measurement of the growth curves (Maker's fringe) of harmonic generation in an atomic gas cell has been achieved by translating the exit window of the gas cell. The data provided direct characterization of the effects of absorption and phase matching on the efficiency of high harmonic generation. ${ }^{12}$ However, the gas-cell technique cannot be used in a cluster jet. In this Letter, we present a tomographic technique for studying high harmonic generation in cluster jets. The technique is based on the programming of plasma density distribution by laser machining. ${ }^{13}$ The growth of harmonic energy as a function of the interaction length was resolved by scanning the end of the cluster density distribution in the path of the pump pulse. A downstream shift of the starting position of rapid growth and a decrease of the slope with increasing backing pressure were observed. These effects yield an optimal backing pressure for high harmonic generation in argon cluster jets.

A $10 \mathrm{TW}, 45 \mathrm{fs}, 810 \mathrm{~nm}$, and $10 \mathrm{~Hz}$ Ti:sapphire laser system based on chirped-pulse amplification (upgraded from the system reported in Ref. 14) was used in this experiment. The linearly polarized laser beam was split in two. One beam served as the pump pulse for driving high-order harmonic generation, and the other, set to be $6 \mathrm{~ns}$ earlier than the pump pulse, was used as the machining pulse. The duration of the 8 mJ pump pulse was set at $180 \mathrm{fs}$ with positive chirp for maximum harmonic production, while the duration of the $30 \mathrm{~mJ}$ machining beam was set at $45 \mathrm{fs}$ for maximum intensity. The setup is shown in Fig. 1. The $8 \mathrm{~mm}$ diameter pump pulse was focused by an f/38 off-axis parabolic mirror onto the center of a cluster jet. The focal spot size of the pump beam was $30 \mu \mathrm{m}$ full width at half-maximum (FWHM) with 90\% energy enclosed in a Gaussian-fit profile, corresponding to a peak intensity of $2.4 \times 10^{15} \mathrm{~W} / \mathrm{cm}^{2}$. Propagating perpendicularly to the pump pulse, the 4 $\mathrm{cm}$ diameter machining pulse was imaged from the location of the knife edge onto the interaction region 


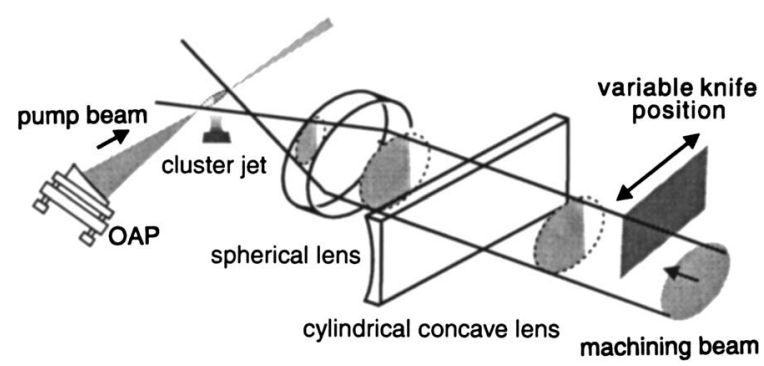

Fig. 1. Setup of the machining beam and the pump beam. OAP, off-axis parabolic mirror.

by a spherical lens of $20 \mathrm{~cm}$ focal length with a demagnification factor of 3 . In the meantime it was focused in the vertical direction to a width of $20 \mu \mathrm{m}$ in FWHM by this spherical lens in combination with a cylindrical concave lens of $75 \mathrm{~cm}$ focal length. This line focus overlapped the propagation path of the pump pulse inside the cluster jet, and the intensity of the machining beam within the overlapped region exceeded the threshold of optical-field ionization. The knife edge, which had a variable transverse position, was used to adjust the region irradiated by the machining beam. The argon cluster jet was produced by using a slit nozzle on a pulsed valve, ${ }^{15}$ and its density profile is a $3.5 \mathrm{~mm}$ flat-top region with $750 \mu \mathrm{m}$ slopes at both edges. The average atom density increased linearly with increasing backing pressure and reached $2.5 \times 10^{19} \mathrm{~cm}^{-3}$ at $3 \times 10^{6} \mathrm{~Pa}$ (400 psi), corresponding to a cluster size of $2.7 \times 10^{6}$ atoms and a cluster radius of $28 \mathrm{~nm}$. In the region that was not blocked by the knife, the machining beam ionized and heated the clusters. After 6 ns the region that was ionized by the machining beam was evacuated as a result of hydrodynamic expansion. By scanning the knife-edge position the end of the interaction region was varied. In this way the growth of harmonic intensity with pump-pulse propagation in the cluster jet was resolved tomographically. Note that such a measurement cannot be accomplished by using a set of gas jet nozzles of various lengths, because the atom density, the cluster size, and the jet profile all change with different nozzles. In that case it will be difficult to vary the interaction length while keeping other parameters fixed.

A flat-field spectrometer consisting of an aperiodic grazing-incident cylindrical grating and a 16-bit $\mathrm{x}$-ray CCD camera was used to measure the $\mathrm{x}$-ray emission spectrum and the divergence angle in the direction of pump-pulse propagation. The spectral range was $17-37 \mathrm{~nm}$. Aluminum filters were used to block transmitted laser pulses and attenuate x-ray emission. By calibrating the grating reflectivity, the filter transmittance, and the CCD response, we obtained the absolute emission yield. The conversion efficiency of the harmonics was $1.5 \times 10^{-7}$ for the 27 th harmonic at $3 \times 10^{5} \mathrm{~Pa}$ (40 psi) backing pressure.

Figure 2 shows the total energy in each harmonic as a function of cluster-jet backing pressure. It was found that there is an optimal cluster-jet backing pressure for maximizing the energy of harmonics that increases with increasing harmonic order, similar to what was observed in an experiment using monomer gas in a hollow fiber. ${ }^{16}$ The optimal backing pressure may be a result of the phase-matching condition or the trade-off between increased gain and increased reabsorption with increasing average atom density.

To determine the main cause for the decrease of harmonic energy at high backing pressures, we used the tomographic technique described above to measure the energy of the 25th harmonic as a function of position in the cluster jet at $1.5 \times 10^{6} \mathrm{~Pa}(200 \mathrm{psi})$ and $3 \times 10^{6} \mathrm{~Pa}$ (400 psi) backing pressures. The results are shown in Fig. 3. By Rayleigh scattering and interferometry, the cluster radii for these backing pressures were measured to be 19 and $28 \mathrm{~nm}$, respectively. ${ }^{17}$ In the figure, the cluster distribution extends from 0 to $5 \mathrm{~mm}$. Along the beam propagation direction the spatial resolution of the tomographic measurement was better than $20 \mu \mathrm{m}$, as shown by the sharpness of side-scattering images in Ref. 13. As shown in the figure, for higher backing pressure significant growth starts at a later position and the slope is smaller. Such a dependence of growth rate on backing pressure leads to a drop-off of overall harmonic production for high backing pressures and thus to the appearance of an optimal backing pres-

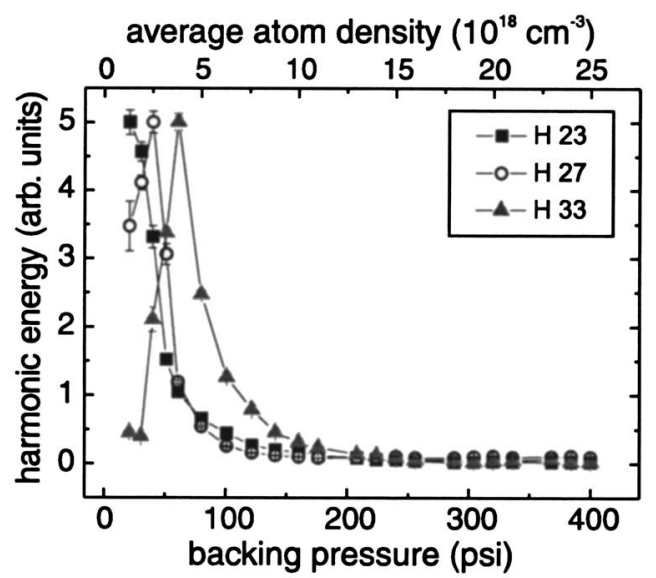

Fig. 2. Energy of the harmonics as a function of cluster-jet backing pressure for various harmonic orders. The peaks are normalized to the same height for viewing convenience.

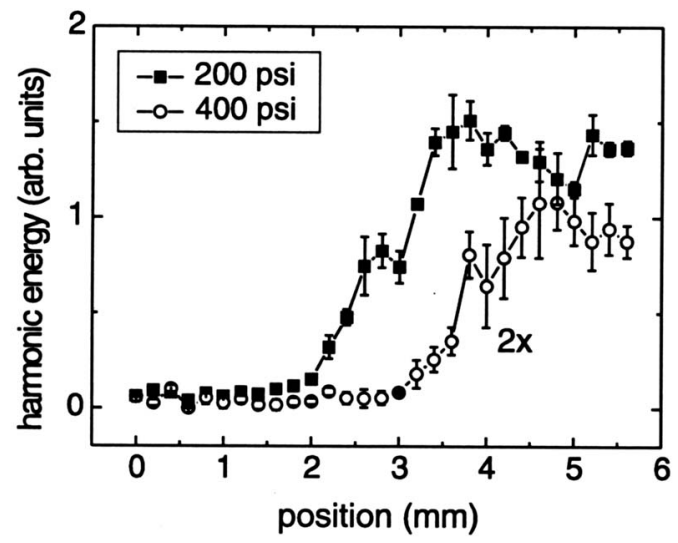

Fig. 3. Energy of the 25th harmonic as a function of position for cluster-jet backing pressures of 200 and 400 psi. The curve for $400 \mathrm{psi}$ is multiplied by 2 for viewing convenience. 


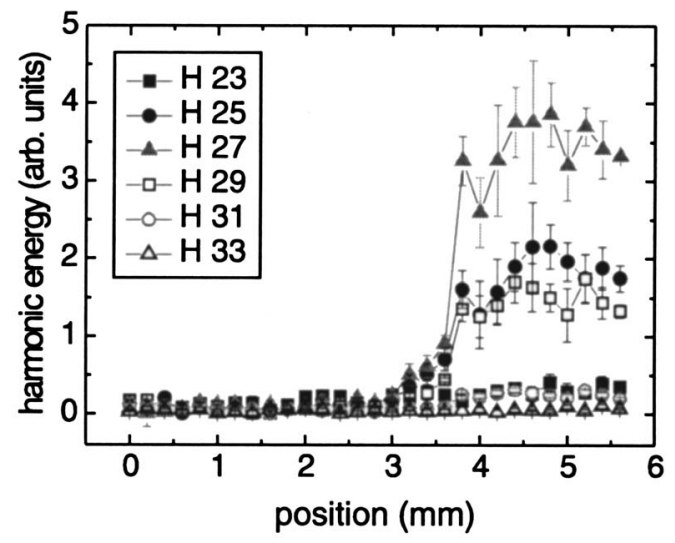

Fig. 4. Energy of the harmonics as a function of position at a cluster-jet backing pressure of 400 psi for various harmonic orders.

sure. In addition, it was also observed that from the 23rd to the 33rd harmonics the regions of fast growth overlap to within $0.4 \mathrm{~mm}$ at both backing pressures of $1.5 \times 10^{6}$ and $3 \times 10^{6} \mathrm{~Pa}$. The latter case is shown in Fig. 4. This indicates that the variations of the positions of fast growth and saturation with respect to backing pressure are not due to larger x-ray reabsorption at higher backing pressure, because the strong dependence of the reabsorption coefficient on harmonic order in this region would make the result sensitive to harmonic order. Therefore, the dominant effect for the observed growth curves and the optimal backing pressure should be the phase-matching condition, which may change significantly for different backing pressures as a result of varying cluster size.

At a backing pressure of $3 \times 10^{5} \mathrm{~Pa}(40 \mathrm{psi}$ ), which is optimal for the production of the 27th and the 29th harmonics, the growth of harmonic energy as a function of the interaction length shows various oscillatory growth behaviors for different harmonic orders. However, at such a low pressure the hydrodynamic expansion of the plasma produced by the machining beam did not evacuate a gas region that was vertically wide enough to cover completely the region of high harmonic generation. As a result, even with the knife edge at zero position the harmonic energy was not zero. This was different from the cases of 1.5 $\times 10^{6}$ and $3 \times 10^{6} \mathrm{~Pa}$. Therefore a vertically wider line focus with sufficient intensity is required for reliable extension of this tomographic technique to the low-density cases. Currently the maximum energy used for the machining pulse is limited by selffocusing and filamentation in the BK7 line-focusing optics and the vacuum window. These limitations can be greatly relieved if low- $n_{2}$ optics made from $\mathrm{CaF}_{2}$ are used.

In summary, by using a tomographic technique based on laser machining we have shown that the growth of high harmonics in a cluster jet as a function of the interaction length can be experimentally resolved. The measurements help to identify the dominant factor that limits the growth of high harmonics. The technique demonstrated in this Letter can thus provide the information required for designing various schemes for phase-matching and quasiphase-matching of high-order harmonic generation in a cluster jet.

This work was partly supported by National Science Council of Taiwan grant NSC 94-2112-M-001010. S.-Y. Chen's e-mail address is sychen @ltl.iams.sinica.edu.tw.

\section{References}

1. T. Brabec and F. Krausz, Rev. Mod. Phys. 72, 545 (2000).

2. J. Seres, E. Seres, A. J. Verhoef, G. Tempea, C. Streli, P. Wobrauschek, V. Yakovlev, A. Scrinzi, C. Spielmann, and F. Krausz, Nature 433, 596 (2005).

3. M. Hentsche, R. Kienberger, Ch. Spielmann, G. A. Reider, N. Milosevic, T. Brabec, P. Corkum, U. Heinzmann, M. Drescher, and F. Krausz, Nature 414, 509 (2001).

4. T. D. Donnelly, T. Ditmire, K. Neuman, M. D. Perry, and R. W. Falcone, Phys. Rev. Lett. 76, 2472 (1996).

5. J. W. G. Tisch, T. Ditmire, D. J. Frasery, N. Hay, M. B. Mason, E. Springate, J. P. Marangos, and M. H. R. Hutchinson, J. Phys. B 30, L709 (1997).

6. C. Vozzi, M. Nisoli, J-P. Caumes, G. Sansone, S. Stagira, S. De Silvestri, M. Vecchiocattivi, D. Bassi, M. Pascolini, L. Poletto, P. Villoresi, and G. Tondello, Appl. Phys. Lett. 86, 111121 (2005).

7. S. X. Hu and Z. Z. Xu, Appl. Phys. Lett. 71, 2605 (1997).

8. V. Véniard, R. Taïeb, and A. Maquet, Phys. Rev. A 65, 013202 (2001).

9. J. R. Vázquez de Aldana and L. Roso, J. Opt. Soc. Am. B 18, 325 (2001)

10. T. Tajima, Y. Kishimoto, and M. C. Downer, Phys. Plasmas 6, 3759 (1999).

11. J. W. G. Tisch, Phys. Rev. A 62, 041802(R) (2000).

12. S. Kazamias, D. Douillet, F. Weihe, C. Valentin, A. Rousse, S. Sebban, G. Grillon, F. Augé, D. Hulin, and Ph. Balcou, Phys. Rev. Lett. 90, 193901 (2003).

13. C.-H. Pai, S.-Y. Huang, C.-C. Kuo, M.-W. Lin, J. Wang, S.-Y. Chen, C.-H. Lee, and J.-Y. Lin, Phys. Plasmas 12, 070707 (2005).

14. H.-H. Chu, S.-Y. Huang, L.-S. Yang, T.-Y. Chien, Y.-F. Xiao, J.-Y. Lin, C.-H. Lee, S.-Y. Chen, J. Wang, Appl. Phys. B 79, 193 (2004).

15. H. H. Chu, H.-E. Tsai, Y.-F. Xiao, C.-H. Lee, J.-Y. Lin, J. Wang, and S.-Y. Chen, Phys. Rev. E 69, 035403(R) (2004).

16. C. G. Durfee III, A. R. Rundquist, S. Backus, C. Herne, M. M. Murnane, and H. C. Kapteyn, Phys. Rev. Lett. 83, 2187 (1999).

17. K. Y. Kim, V. Kumarappan, and H. M. Milchberg, Appl. Phys. Lett. 83, 3210 (2003). 